\title{
Escoliosis idiopática
}

\author{
LI. Álvarez García de Quesadaa ${ }^{a}$ A. Núñez Giralda ${ }^{b}$ \\ ${ }^{a}$ Consultor en Cirugía Ortopédica y Traumatología. Unidad de Columna. \\ Hospital Universitario Quirón. Madrid. España. \\ bPediatra. CS Guayaba. Madrid. España.
}

\section{Resumen}

La escoliosis idiopática es motivo de consulta tanto en Atención Primaria como en Atención Especializada, por las connotaciones que supone el tratamiento tanto ortopédico como quirúrgico. Se define como la deformidad de la columna vertebral en el plano anteroposterior superior a $10^{\circ}$. Tiene una prevalencia aproximada del $2 \%$. Los estudios más recientes hablan a favor de una predisposición genética en el desarrollo de las curvas. Los cribados para su diagnóstico se han demostrado caros e ineficaces. El adecuado conocimiento de una exploración básica y de la historia natural de la enfermedad evita la exposición radiológica excesiva. La escoliosis idiopática más frecuente es la del adolescente, por lo que hay que prestar especial atención en la exploración a los niños de 10-11 años. El tratamiento está muy estandarizado, teniendo en cuenta el tipo y la magnitud de la curva, el sexo y el estado madurativo del paciente. La indicación del corsé es para las curvas inmaduras de 25 a $45^{\circ}$ y la cirugía para las que superen los $45^{\circ}$. Los actuales sistemas de instrumentación hacen innecesario esperar a la edad madura para realizar la cirugía.

Palabras clave: Escoliosis idiopática. Cribado.

\section{Idiopathic scoliosis}

\section{Abstract}

Idiopathic scoliosis is a reason for consultation in both Primary Care and in Specialized Care because of the connotations that implies both orthopaedic and surgical treatment. It is defined as the coronal plane deformity greater than 10 degrees. It has a prevalence of approximately $2 \%$. The most recent studies speak in favour of a genetic predisposition in the development of the curves. The scoliosis screening programs for diagnosis have proved expensive and ineffective. An adequate knowledge of basic exploration and natural history of the disease prevents excessive radiation exposure to children. Idiopathic scoliosis is most common in the adolescent. We need to pay special attention to the exploration of children aged 10-11. The treatment is very standardized, taking into account the type and magnitude of the curve, sex and skeletal maturity of the patient. Given these factors, an indication of the back brace is for immature curves 25 to $45^{\circ}$ and surgery for curves that exceed 45 degrees. Current instrumentation systems allow surgery before skeletal maturity.

Key words: Idiopathic scoliosis. Screening.

L. Ignacio Álvarez García de Quesada, ialvarez.mad@quiron.es

Los autores declaran no presentar conflictos de intereses en relación con la preparación y publicación de este artículo. 


\section{Introducción}

La escoliosis es la deformidad del esqueleto axial en el plano anteroposterior. Incluye deformidad tridimensional con rotación vertebral y puede acompañarse de alteraciones en el plano sagital. Para que se considere escoliosis debe tener más de $10^{\circ}$ de angulación.

Se define como idiopática cuando se han descartado otras causas (neuromuscular, congénita, tumoral, infecciosa, traumática o sindrómica). El término idiopático puede terminar por ser inadecuado, dado que los estudios actuales indican que la escoliosis idiopática del adolescente es una enfermedad poligénica con varios patrones de herencia ${ }^{1}$. Los marcadores genéticos que se han identificado están relacionados con los que suponen un mayor riesgo de progresión de la curva y que llegan a un nivel de gravedad tal que precise de cirugía. Estos marcadores genéticos han sido validados en niñas y niños caucásicos, pero aún no están confirmados en los asiáticos o afroamericanos. Proporcionan una base para calcular el riesgo de progresión de la escoliosis, que permite personalizar las decisiones terapéuticas.

La prevalencia de la escoliosis idiopática se aproxima al $2 \%$ de la población, con una frecuencia cinco veces mayor en niñas que en niños².
Un correcto conocimiento de la enfermedad no solo reduce los costes económicos del diagnóstico y del tratamiento sino también, y lo más importante, la sobreexposición radiológica, en muchos casos innecesaria. Se ha relacionado con un aumento de la incidencia de cáncer de mama en niñas con escoliosis ${ }^{3}$.

\section{Clasificación}

La escoliosis idiopática se clasifica por la edad de aparición, siendo infantil cuando aparece desde el nacimiento hasta los tres años, juvenil cuando aparece desde los tres años hasta los diez (pubertad), y del adolescente cuando aparece después de los diez años.

La escoliosis infantil es rara, con un porcentaje del 0,5 al $5 \%$ de las escoliosis, la infantil del 7 al 10,5\% y la del adolescente, la más frecuente, con el $89 \%$ de los casos.

\section{Etiología}

Se han valorado diferentes causas como origen de la escoliosis idiopática, desde alteraciones en el crecimiento, alteraciones discales, alteraciones del sistema nervioso central, hasta anomalías en el tejido conectivo y desequilibrios musculares. Ninguna se ha confirmado.

En las últimas décadas ha tomado mayor fuerza la teoría genética, con una he- 
rencia poligénica. En los trabajos de Ogilvie $^{4}$ se analizan 200 marcadores genéti$\cos$, y encuentra que cuanta mayor presencia tienen, más gravedad y posibilidad de progresión de las curvas existe.

\section{Clínica}

La escoliosis no es dolorosa. La prevalencia de dolor de espalda en el adolescente se aproxima al de la edad adulta ${ }^{5}$, por lo que no debe relacionarse con la escoliosis.

En un paciente con escoliosis y dolor de espalda, debe ampliarse el estudio para descartar otro tipo de lesiones en su esqueleto axial (hernias discales, siringomielia, médula anclada, infecciones, tumores, etc.).

El motivo de consulta en Traumatología suele ser por presentar el niño/niña una deformidad, que ha sido detectada por sus padres, en la revisión realizada por su pediatra o en el colegio. Hay que señalar que los programas de detección de la escoliosis en los adolescentes se han considerado $\operatorname{costosos}^{6}$ y poco eficaces, al tener protocolos dispares entre ellos?.

Aunque la incidencia global de escoliosis es del $2 \%$, la escoliosis de interés, por su necesidad de tratamiento (las curvas que superan los $25^{\circ}$ ), se reduce al 2-3/1000.

\section{Evaluación y manejo del paciente}

El objetivo fundamental es identificar los casos que necesitarán tratamiento, lo más precozmente y con la menor exposición radiológica. Lo más fácil para diagnosticar la escoliosis es con una radiografía de columna completa, pero está contraindicado realizarla de forma rutinaria.

Dado que la escoliosis idiopática es más frecuente en la adolescencia, debe hacerse una evaluación clínica alrededor de los 10-11 años (antes de la menarquia).

La exploración incluye una exploración clínica general y una evaluación del paciente en bipedestación, para valorar la alineación global de la columna en el plano sagital (lateral) y coronal (anteroposterior). Valorar asimetrías en los hombros, escápulas y flancos. Comprobar si existen discrepancias en la longitud de los miembros inferiores. Realizar una exploración neurológica básica y buscar otro tipo de deformidades: cavismo de pies, etc.

La exploración de cribado más simple y conocida, y que se aplica en Atención Primaria, es el test de Adams ${ }^{8}$. Valora la asimetría del tronco desde detrás, con el niño flexionado hacia delante. Se considera positivo cuando el torso del niño no está completamente paralelo al suelo, sino que presenta una giba a ni- 
vel dorsal o deformidad lumbar. Un test de Adams positivo significa que el paciente presenta una rotación en el tronco y una posible escoliosis. Clásicamente, un test de Adams positivo era indicación de estudio radiográfico, pero hoy día intentamos ajustar más las indicaciones. Para ello, se emplea un inclinómetro o escoliómetro de Bunnell, que indica o mide la inclinación del tronco (figura 1). Si no se dispone de un inclinómetro, el niño con un test de Adams positivo debe ser remitido a la consulta especializada para su valoración. Insistimos en que aún no debe pedirse la radiografía.
Si se dispone de un inclinómetro, la pauta indicada es la siguiente: con un Adams positivo y menos de $5^{\circ}$ de escoliómetro (figura 2), podemos afirmar que no tiene escoliosis (o al menos no es significativa); si el escoliómetro se encuentra entre los 5 y los $9^{\circ}$, ese niño debe ser reevaluado pasados seis meses (no hay indicación de radiografía) y realizar el seguimiento hasta un año después de la menarquia; finalmente, si el niño presenta $10^{\circ}$ o más en el escoliómetro (figura 3), debe realizarse una telerradiografía anteroposterior de columna completa en bipedestación y remitir al especialista.

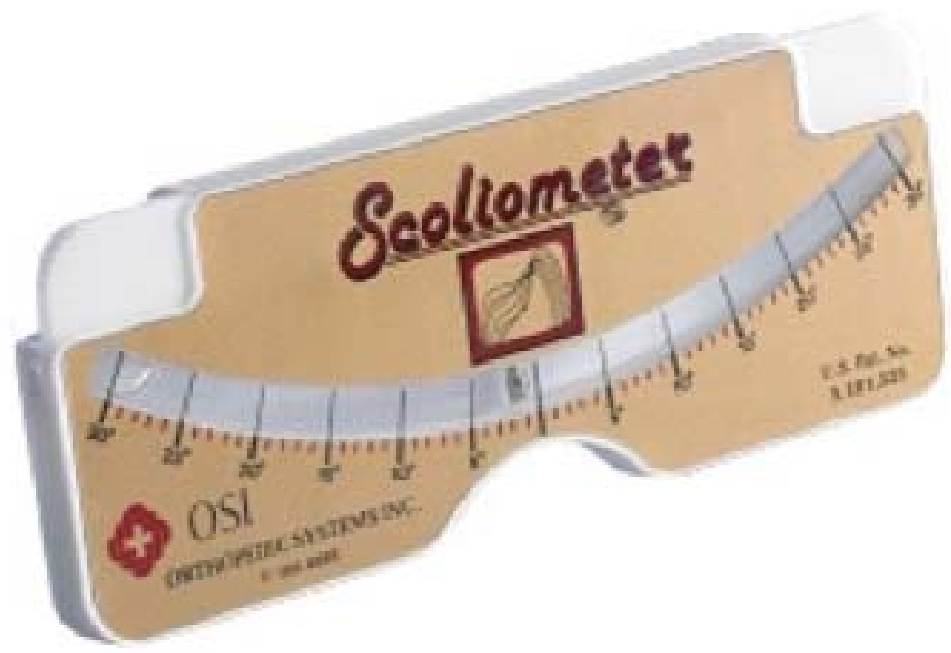


Figura 2. Test de Adams negativo; escoliómetro con $0^{\circ}$ de inclinación.

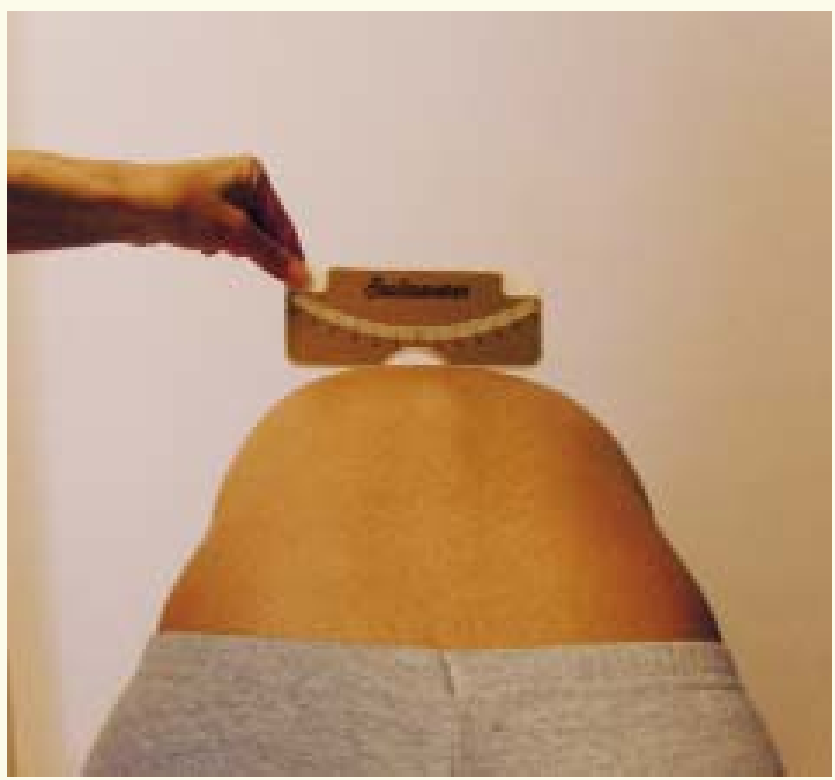

Figura 3. Test de Adams positivo; escoliómetría patológica ( $18^{\circ}$ de inclinación del tronco).

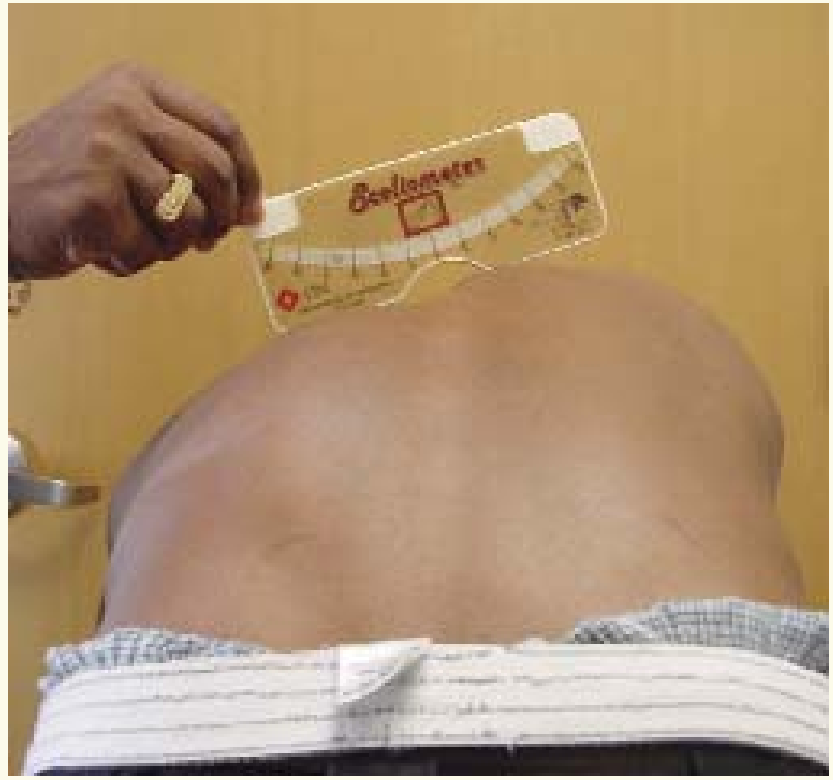


Con el estudio radiográfico confirmamos que tiene una curva con un ángulo de Cobb mayor de $10^{\circ}$ (un ángulo de inclinación con el escoliómetro de más de $10^{\circ}$ supone una curva aproximada de $15-20^{\circ}$ de Cobb).

\section{Evaluación radiográfica}

En el estudio radiográfico se puede valorar la magnitud, el tipo de la curva y el estado madurativo del esqueleto axial.

La magnitud de la curva se calcula con la medida del ángulo de Cobb, que es el ángulo que forman las vértebras más inclinadas en la parte superior e inferior de la curva (figura 4). Se traza la perpendicular al platillo superior de la vértebra limitante superior y la perpendicular al platillo inferior de la vértebra limitante inferior, Donde se cruzan ambas líneas se mide el ángulo de Cobb.

El tipo de curva se clasifica según la localización del ápex de la curva (vértebra más alejada de la línea media) en: torácicas, toracolumbares y lumbares. Es posible la presencia de dobles curvas. La clasificación que actualmente se emplea para determinar el tratamiento más adecuado de la escoliosis idiopática del adolescente es la clasificación de Lenke ${ }^{9}$ (figura 5). que valora tanto las curvas como su flexibilidad, medida por los cambios detecta- 
Figura 5. La clasificación que actualmente se emplea para determinar el tratamiento más adecuado de la escoliosis idiopática del adolescente es la clasificación de Lenke9.

\begin{tabular}{|c|c|c|c|c|}
\hline \multicolumn{5}{|c|}{ Curve Type } \\
\hline Type & $\begin{array}{l}\text { Proximal } \\
\text { Thoracic }\end{array}$ & $\begin{array}{c}\text { Maln } \\
\text { Thoracic }\end{array}$ & $\begin{array}{c}\text { Thoracolumbar I } \\
\text { Lumbar }\end{array}$ & $\begin{array}{l}\text { Curve } \\
\text { Type }\end{array}$ \\
\hline 1 & Non-Structural & Structural (Major") & Non-Structural & Main Thoracic (MT) \\
\hline 2 & Structural & Structural (Major") & Non-Structural & Double Thoracic (DT) \\
\hline 3 & Non-Structural & Structural (Major") & Structural & Double Major (OM) \\
\hline 4 & Structural & Structural (Major") & Structural & Triple Major (TM) \\
\hline 5 & Non-Structural & Non-Structural & Structural (Major") & Thoracolumbar / Lumbar (TLL) \\
\hline 6 & Non-Structural & Structural & Structural (Major") & $\begin{array}{l}\text { Thoracolumbar / Lumbar. } \\
\text { Main Thoracic (TLU - MT) }\end{array}$ \\
\hline
\end{tabular}

STRUCTURAL CRITERIA

(Minor Curves)

Proximal Thoracic - Side Bending Cobb $225^{\circ}$ - T2 - T5 Kyphosis $\geq+20^{\circ}$

Main Thoracic - Side Bending Cobb $\geq 25^{\circ}$ Thoracolumber / Lumbar. - Side Bending Cobb $\geq 25^{\circ}$ - T10 - L2 Kyphosis $z+20^{\circ}$
-Major = Largest Cobb Measurement, always structural Minor = all other curves with structural criteria applied

\begin{tabular}{|c|c|}
\hline \multicolumn{2}{|c|}{ LOCATION OF APEX } \\
\hline$\frac{\text { CURVE }}{\text { THORACIC }}$ & $\mathrm{T} 2_{2} \frac{\mathrm{APEX}}{\mathrm{T} 11.12 \text { DISC }}$ \\
\hline THORACOLUMBAR & T12 - L1 \\
\hline LUMBAR & L1-2 DISC - L4 \\
\hline
\end{tabular}

\begin{tabular}{|c|c|c|c|c|c|c|c|}
\hline \multicolumn{5}{|c|}{ Modifiers } & \multirow{2}{*}{\multicolumn{2}{|c|}{$\begin{array}{c}\text { Thoracic Sagittal } \\
\text { Profile } \\
\text { T5 - T12 }\end{array}$}} & \\
\hline $\begin{array}{l}\text { Lumbar } \\
\text { Splne } \\
\text { Modifier }\end{array}$ & $\begin{array}{c}\text { CSVL to Lumber } \\
\text { Apex }\end{array}$ & \multirow{4}{*}{\multicolumn{3}{|c|}{ A }} & & & \\
\hline A & CSVL Between Pedicles & & & & $=$ & (Hypo) & $<10^{\circ}$ \\
\hline B & $\begin{array}{c}\text { CSVL Touches Apical } \\
\text { Body(ies) }\end{array}$ & & & & N & (Normal) & $10^{\circ} \cdot 40^{\circ}$ \\
\hline C & CSVL Completely Medial & & & & + & (Hyper) & $>40^{\circ}$ \\
\hline
\end{tabular}

Curve Type (1.6) + Lumbar Spine Modifier (A, B, or C) + Thoracic Sagittal Modifier ( N s. -) Classification (e.g.1B ):

Figura 6. La madurez esquelética se determina radiológicamente por el signo de Risser, estado de madurez del cartílago de crecimiento de la cresta ilíaca.

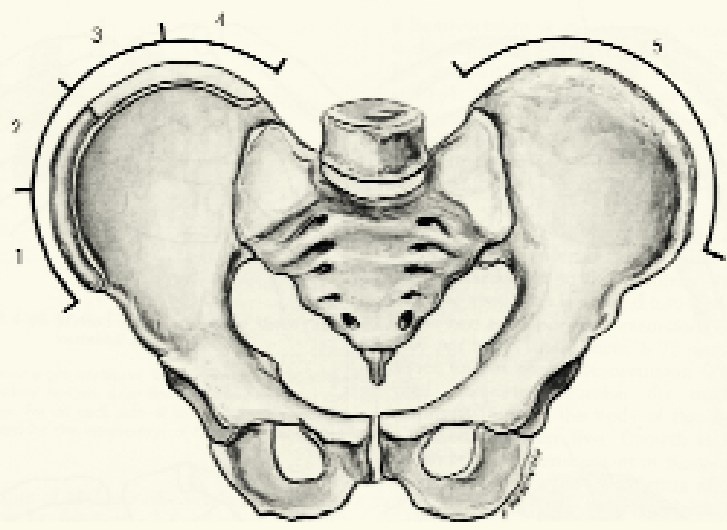


dos en las radiografías dinámicas en flexión lateral (bending test).

La madurez esquelética se determina radiológicamente por el signo de Risser, estado de madurez del cartílago de crecimiento de la cresta ilíaca (figura 6). Cuando no hay osificación está en el estadio 0. Con la madurez, la osificación avanza desde la espina ilíaca anterosuperior hasta la posterior, y se divide en estadios de madurez, dividiendo la cresta en cuartos. En el primer cuarto estaría el estadio 1, hasta la mitad el estadio 2, entre la mitad y tres cuartos, el estadio 3 y hasta el final del estadio 4 . Cuando ha madurado completamente (ya no se visualiza la línea madurativa y la pelvis se ha osificado completamente), es el estadio Risser 5.

\section{Historia natural}

Un hecho importante en el tratamiento de la escoliosis es la historia natural, conocer en qué casos progresa. Los factores que predicen la posibilidad de progresión de las curvas son la edad, el sexo, el estado puberal (Tanner), la madurez ósea (Risser), la localización de la curva y la magnitud de la curva en el momento del diagnóstico.

El factor que se ha demostrado más importante en la progresión de la escoliosis es la magnitud de la curva en el momento del diagnóstico ${ }^{10}$. Un ángulo de Cobb al diagnóstico de $25^{\circ}$ o mayor tiene un $68,4 \%$ de posibilidades de progresión hasta los $30^{\circ}$ o más, en la madurez esquelética. Por el contrario, curvas con un ángulo de Cobb menor de $25^{\circ}$ tienen un $91,9 \%$ de posibilidades de no progresar. El caso más típico es el de una niña menor de 12 años con un Cobb de $25^{\circ}$ o más, que tiene un $82 \%$ de posibilidades de progresar. Mientras que un niño postpuberal, de 12 años o más, con menos de $25^{\circ}$ al diagnóstico, solo tiene un $2,4 \%$ de posibilidades de progresar.

Hay diferencias en el riesgo en cuanto al sexo y a la madurez ósea. En la tabla 1 se valora la posibilidad de progresión según la madurez ósea y en la tabla 2 en relación con la pubertad y la magnitud de la curva al diagnóstico.

A largo plazo, una escoliosis sin tratar puede ocasionar dolor de espalda, con una incidencia mayor que en la población normal; deterioro de la función respiratoria, en el caso de las curvas torácicas; deformidad estética, que puede ser progresiva, sobre todo en curvas de más de $50^{\circ}$, y mayor índice de mortalidad ${ }^{10}$.

\section{Tratamiento de la escoliosis}

Una vez valorado qué curvas tienen más riesgo de progresar, las indicaciones 
Tabla 1. Posibilidad de progresión en cuanto a la madurez ósea

\begin{tabular}{c|c|c}
\hline Signo de Risser & Curva de $\mathbf{5}$ a $\mathbf{1 9}^{\circ}$ & Curva de 20 a 29 \\
\hline $0-1$ & $22 \%$ & $68 \%$ \\
$2-4$ & $1,6 \%$ & $23 \%$ \\
\hline
\end{tabular}

Tabla 2. Posibilidad de progresión en relación con la pubertad y la magnitud de la curva al diagnóstico

\begin{tabular}{|c|c|c|c|c|}
\hline Sexo & Pubertad & $\begin{array}{c}\text { Ángulo } \\
\text { al inicio }>25^{\circ}\end{array}$ & $\begin{array}{c}\text { Edad al } \\
\text { diagnóstico } \\
<12 \text { años }\end{array}$ & $\begin{array}{c}\text { Probabilidad } \\
\text { superar } 30^{\circ}\end{array}$ \\
\hline \multirow[t]{8}{*}{ Varón } & \multirow[t]{4}{*}{ Sí } & \multirow[t]{2}{*}{ No } & No & 2,39 \\
\hline & & & Sí & 2,83 \\
\hline & & \multirow[t]{2}{*}{ Sí } & No & 40,25 \\
\hline & & & Sí & 44,42 \\
\hline & \multirow[t]{4}{*}{ No } & \multirow[t]{2}{*}{ No } & No & 5,30 \\
\hline & & & Sí & 6,22 \\
\hline & & \multirow[t]{2}{*}{ Sí } & No & 60,59 \\
\hline & & & Sí & 64,59 \\
\hline \multirow[t]{8}{*}{ Mujer } & \multirow[t]{4}{*}{ Sí } & \multirow[t]{2}{*}{ No } & No & 5,85 \\
\hline & & & Sí & 6,87 \\
\hline & & \multirow[t]{2}{*}{ Sí } & No & 63,09 \\
\hline & & & Sí & 66,97 \\
\hline & \multirow[t]{4}{*}{ No } & \multirow[t]{2}{*}{ No } & No & 12,42 \\
\hline & & & Sí & 14,41 \\
\hline & & \multirow[t]{2}{*}{ Sí } & No & 79,59 \\
\hline & & & Sí & 82,23 \\
\hline
\end{tabular}

del corsé serían las siguientes: curvas de 30-40 con un Risser de 0-2, curvas entre 20 y $29^{\circ}$, pero que han sufrido una progresión mayor de $5^{\circ}$ en el último año, y curvas de $25-29^{\circ}$ con un Risser 0 y un Tanner 1-2. No está indicado en curvas de más de $45^{\circ}$ (indicada la cirugía), o menores de $25^{\circ}$ que no tienen progresión. Con Risser de 3 o más, no está indicado, por el muy bajo riesgo de progresión ${ }^{11}$.

Hay diferentes tipos de corsés, cuya indicación varía en virtud del ápex de la curva, las condiciones del paciente y la duración del tratamiento durante el día (23 horas o nocturnos) $)^{12,13}$.

Las indicaciones de cirugía son todas las curvas de más de $45^{\circ}$ en niñas premenárquicas con Risser 0 y Taner 2-3. Así mismo, las curvas de $40-45^{\circ}$ que presenten progresión a pesar del corsé, y en el adolescente maduro con curva mayor de $50^{\circ}$. En la figura 7 se muestra el algoritmo de tratamiento de la escoliosis. La corrección de la deformidad se consigue con cirugía. Generalmente, se realiza mediante la colocación de torni- 
Figura 7. Algoritmo de tratamiento de la escoliosis.

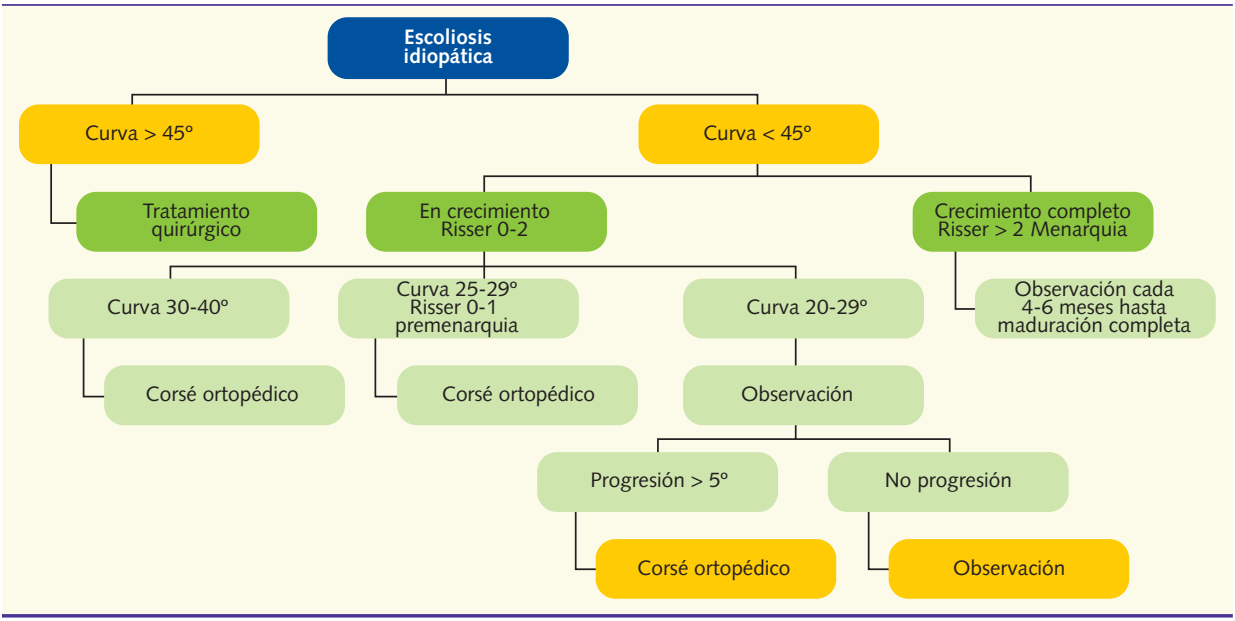

Figura 8. Imagen preoperatoria; niña de 15 años con escoliosis idiopática del adolescente.

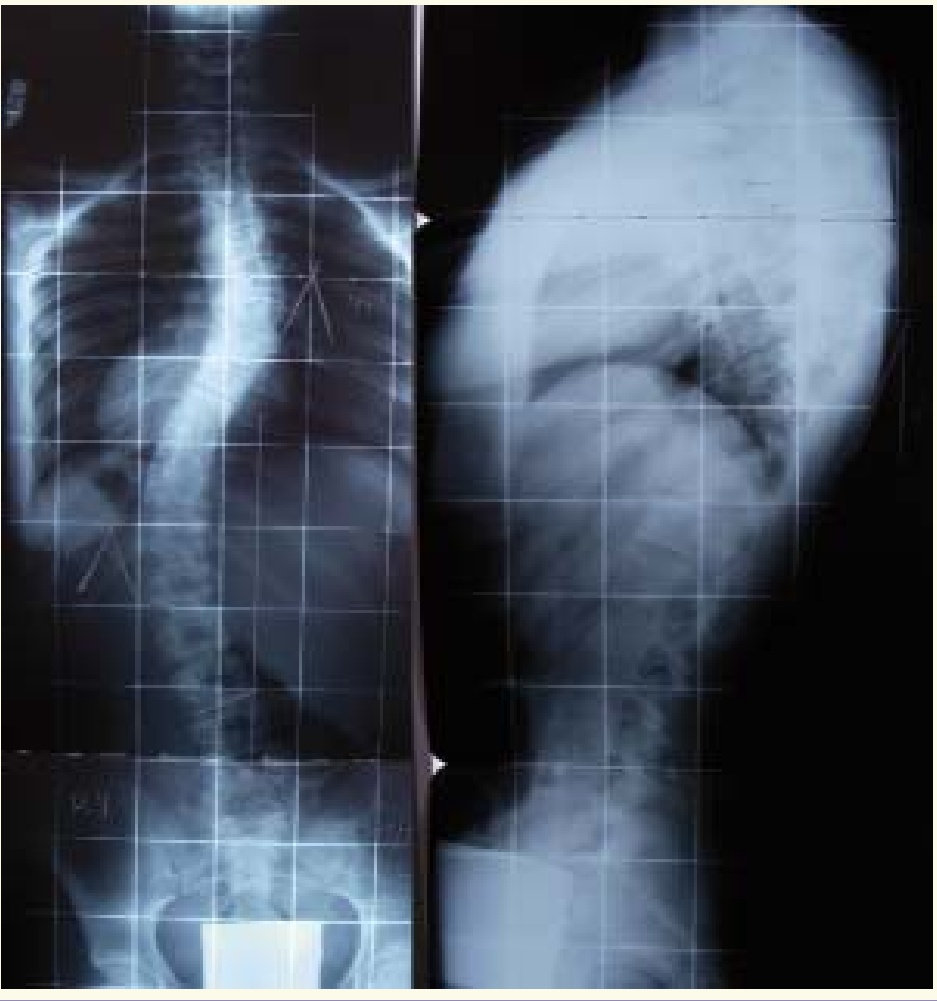


Figura 9. Imagen postoperatoria de la paciente de la figura anterior.

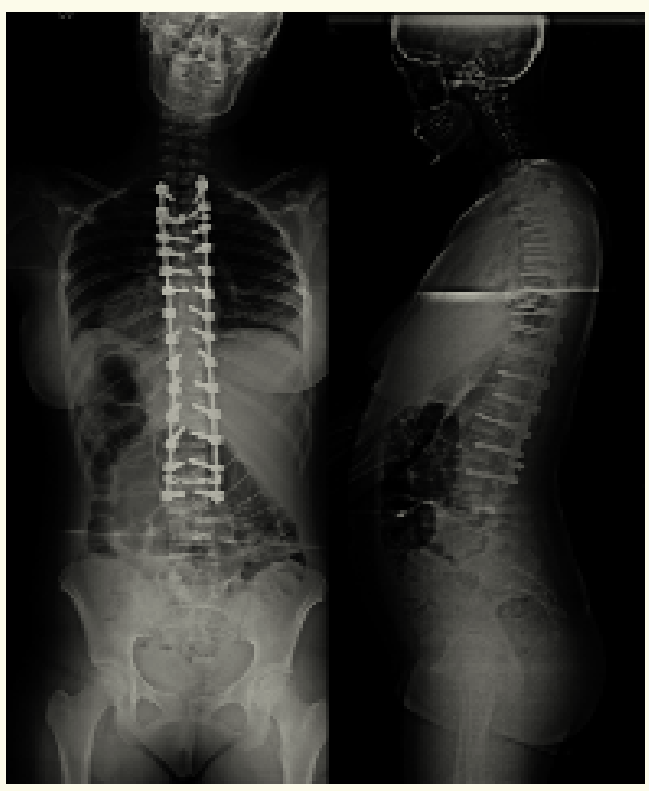

llos y barras en las vértebras implicadas en la deformidad, y con diferentes instrumentales se realiza la realineación (figuras 8 y 9). Se han mejorado las instrumentaciones en cirugía de columna y se consiguen mejores tasas de corrección y más anatómicas. También, la introducción de la monitorización neurofisiológica intraoperatoria ha añadido mayor seguridad durante las intervenciones.

\section{Bibliografía}

1. Ogilvie J, Braun J, Argyle V, Nelson L, Meade M, Waard K. The search for Idiopathic Scoliosis Genes. Spine. 2006;31:679-81.

2. Rogala EJ, Drummond DS, Gurr J. Scoliosis incidence and natural history: a prospective epidemiological study. J Bone Joint Surg Am. 1976;60:173-6.

3. Doody MM, Lonstein JE, Stovall $M$, Hacker DG, Luckyanov N, Land CE. Breast Cancer Morta- lity After Diagnostic Radiography. Spine. 2000;25: 2052-63.

4. Ogilvie J. Adolescent idiopathic scoliosis and genetic testing. Curr Opin Pediatr. 2010;22:67-70.

5. Skaggs D, Early S, Ambra P, Tolo V, Kay R. Back Pain and Backpacks in School Children. J Pediatr Orthop. 2006;26:358-63.

6. Lee CF, Fong D, Cheung K, Cheng J, Ng B, Lam $T$ et al. Costs of School Scoliosis Screening. Spine. 2010;35:2266-72. 
7. Bunnell W. Selective Screening for Scoliosis. Clin Orthop. 2005;434:10-45.

8. Grossman TW, Mazur JM, Cummings RJ. An evaluation of the Adams forward bend test and the scoliometer in a scoliosis screening setting. J Pediatr Orthop. 1995;15:535-8.

9. Lenke LG, Betz RB, Harms J. Adolescent idiopathic scoliosis: a new classification to determine extent of spinal arthrodesis. J Bone Joint Surg Am. 2001;83:1169-81.

10. Tan K, Moe MM, Vaithinathan R, Wong HK. Curve Progression in Idiopathic Scoliosis. Follow-up Study to Skeletal Maturity. Spine. 2009; 34:697-700.
11. Grabowski G, Gelb D. Classification and treatment of adolescent idiophatic scoliosis. Curr Opin Orthop. 2005;16:158-62.

12. Gammon S, Mehlman C, Chan W, Heifetz J, Durrett G, Wall E. A Comparison of thoracolumbosacral Orthoses and SpineCor Treatment of Adolescent Idiopathic Scoliosis Patients Using the Scoliosis Research Society Standardized Criteria. J Pediatr Orthop. 2010;30:531-8.

13. Clin J, Aubin CE, Parent S, Labelle H. A Biomechanical Study of the Charleston Brace for the Treatment of Scoliosis. Spine. 2010;35:940-7. 